\title{
NOVO DESIGN PARA A REDE FEDERAL DE EDUCAÇÃO TECNOLÓGICA
}

\author{
Francisco das Chagas de Mariz Fernandes \\ Professor do Departamento de Gestão do CEFET-RN, Mestre em Engenharia de Produção. \\ mariz@cefetrn.br
}

\section{RESUMO}

A partir do ano de 2003, o Governo Lula iniciou ações voltadas à democratização da oferta da educação técnica no país, através de um plano estruturante de expansão da Rede Federal de Educação Profissional e Tecnológica. A primeira fase do plano compreendeu a construção de 64 novas Unidades de Ensino para somar às 140 que já existiam. Ao mesmo tempo, o Ministério da Educação logo deu início à segunda fase do projeto, para a implantação de mais 150 novas Escolas, devendo totalizar, até 2010, a meta de 354 instituições. Nesse contexto, surge a proposta de constituição de 38 Institutos Federais de Educação, Ciência e Tecnologia para integrar, a partir do exercício de 2009, essas Unidades de Ensino, bem como constituir a Rede Federal de Educação Profissional, Científica e Tecnológica. Cada Instituto Federal é estruturado com uma Reitoria e vários Campi, com gestão interdependente entre ambos. Territorialmente, à Reitoria competirá a função estratégica de definição de políticas, supervisão e controle. Para tanto, necessita de uma estrutura administrativa que congregue, além do gabinete, pró-reitorias e diretorias de atuação sistêmica, cabendo a esses órgãos a função de trabalhar matricialmente vinculados às unidades afins dos Campi. Cada Campus, por sua vez, responsável pela execução dos objetivos finalísticos institucionais, necessita de uma estruturação híbrida, através da associação da departamentalização funcional e a matricial - para viabilizar o diálogo e interação dos departamentos da área acadêmica com as unidades operacionais dos demais departamentos das áreas de administração, de apoio ao ensino, de extensão e de pesquisa. $\mathrm{Na}$ realidade, o arranjo situacional dos Institutos Federais é novo e caracteriza-se como próprio de uma estrutura em rede, para integrar sistemicamente diversas organizações de ensino através de um núcleo central - no âmbito nacional, através da Secretaria de Educação Profissional e Tecnológica do Ministério da Educação, e no espaço regional, os vários Campi, através de uma Reitoria.

PALAVRAS-CHAVE: Educação Profissional; Expansão; Rede Federal; Instituto Federal.

\section{NEW DESIGN FOR FEDERAL NETWORK OF TECHNOLOGICAL EDUCATION}

\begin{abstract}
Since the year of 2003, Lula's Government initiated actions directed to the democratization of technique education offer in the country, through a structuring plan of expansion of Federal Network of Professional and Technological Education. The first phase of the plan embraced the construction of 64 (sixty-four) new Education Units to sum up to the 140 (one hundred forty) that already existed. At the same time, the Ministry of Education started second phase of the Project, for the implantation of more 150 (one hundred fifty) new Schools, totalizing, up to 2010, the goal of 354 (three hundred and fifty-four) institutions. In this context, appears the proposal of establishing 38 (thirty-eight) Federal Institutes of Education, Science and Technology to integrate, from 2009, these Education
\end{abstract}


Units, as well as constituting the Federal Network of Professional, Scientific and Technological Education. Each Federal Institute is structured with a Rectory and some campuses, with interdependent management between both. Territorially, to the Rectory will comprehend the strategical function of politics definition, supervision and control. To achieve it, needs an administrative structure that congregates, beyond the cabinet, prorectories and systemic performance directorates, being responsibility of these agencies the function of working matrically entailed to the similar units of the campuses. Each Campus, therefore, responsible for the execution of finalistic institutional objectives, needs a hybrid structuring, through the association of functional and matrical departmentalization - to make possible the dialogue and interaction of the academic area departments with the operational units of the others departments of administration areas, of education support, of extension and research. In reality, the situational arrangement of the Federal Institutes is new and characterized as proper of a network structure, to systematically integrate several organizations of teaching through a central nucleus - in national scope, through the Professional and Technological Education Secretariat of the Ministry of Education, and in regional scope, some Campuses through a Rectory.

KEYWORDS: Professional Education; Expansion; Federal Network; Federal Institute. 


\section{NOVO DESIGN PARA A REDE FEDERAL DE EDUCAÇÃO TECNOLÓGICA}

\section{INTRODUÇÃO}

A partir do ano de 2003, o Governo Lula iniciou ações voltadas à democratização da oferta da educação técnica no país, através de um plano estruturante de expansão da Rede Federal de Educação Profissional e Tecnológica. Até então, o Brasil dispunha de 140 unidades dessas instituições, sendo 34 Centros Federais de Educação Tecnológica, 37 Unidades de Ensino Descentralizadas subordinadas a esses Centros, 36 Escolas Agrotécnicas Federais, uma Escola Técnica Federal e 32 Colégios Técnicos vinculados a Universidades Federais. A primeira fase do plano, em conclusão, compreendeu a construção de 64 novas Unidades de Ensino. Ao mesmo tempo, o Ministério da Educação logo deu início à segunda fase do projeto, para a implantação de mais 150 novas Escolas, devendo totalizar, até 2010, a meta de 354 instituições. Há, ainda, uma expectativa na sociedade brasileira de que, antes do término do Governo Lula, possa ser iniciada a terceira e última fase do plano, contemplando mais 146 unidades de educação profissional e tecnológica, o que resultaria numa rede composta por 500 Escolas estrategicamente disseminadas pelo país, através de cidades-pólos, na condição de referência para os demais sistemas de educação. Dessa forma, busca-se consolidação do amplo projeto de inclusão social do Governo Federal, por meio do fortalecimento da oferta de ensino gratuito de qualidade e de uma formação cidadã.

Essas Escolas, construídas com foco na capacitação e formação profissional e tecnológica de pessoas, através de cursos integrados aos arranjos produtivos, sociais e culturais locais, representam fator decisivo de desenvolvimento nas regiões onde são implantadas. Nas fotos a seguir, tem-se uma visão das Unidades de Currais Novos (figuras 1 e 2) e de Ipanguaçu (figuras 3, 4 e 5), implantadas no Estado do Rio Grande do Norte, durante a primeira fase do projeto de Expansão.

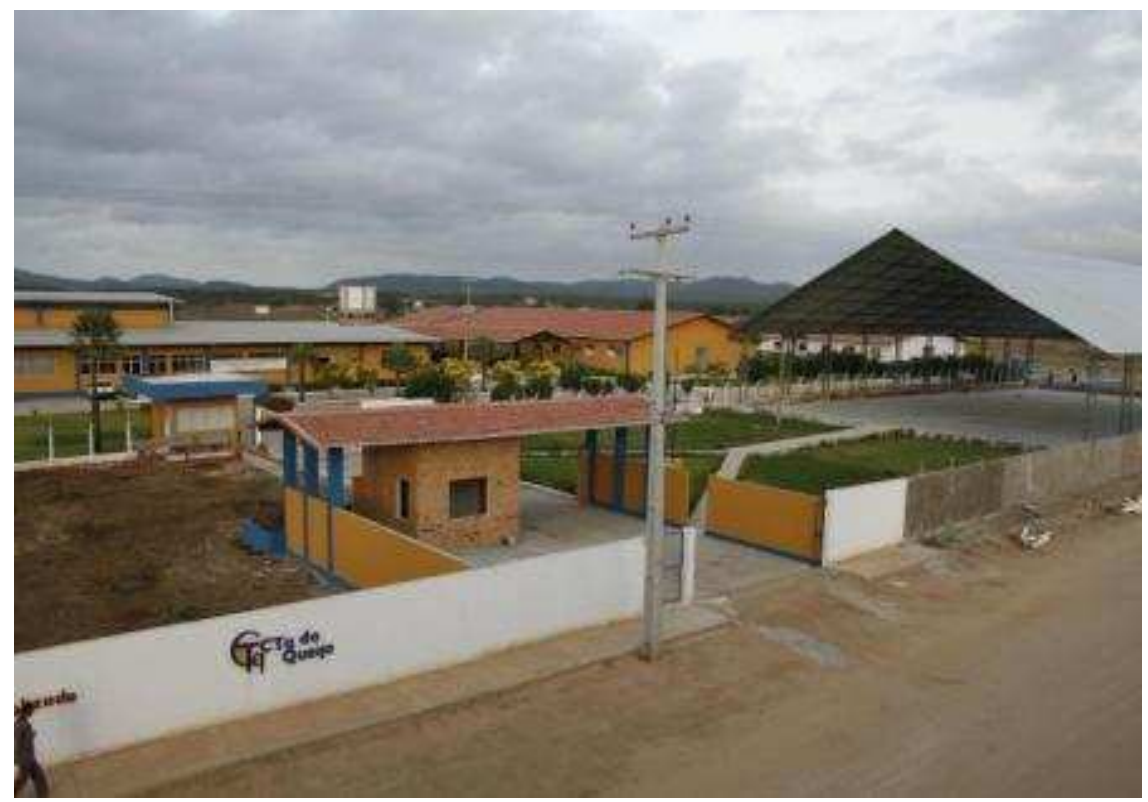

Figura 1 - Vista aérea da Unidade de Ensino de Currais Novos-RN. 


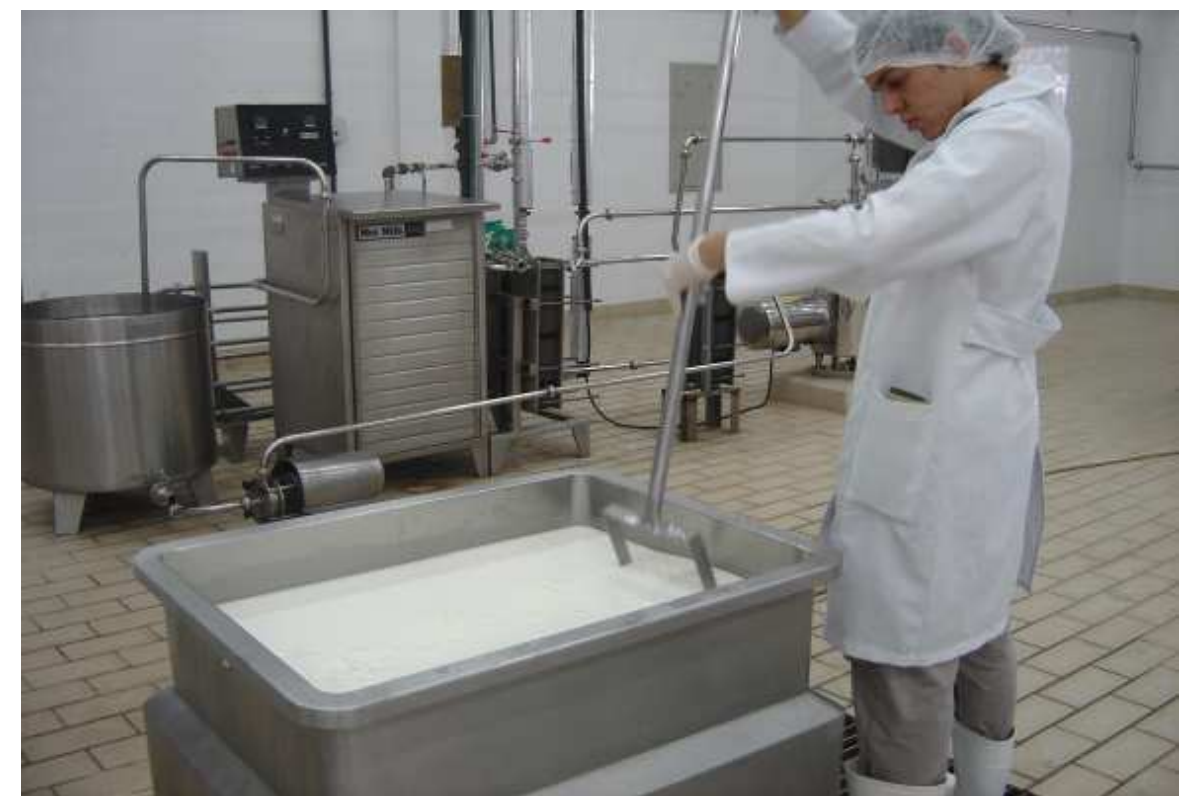

Figura 2 - Instalações do Centro Tecnológico do Queijo - Laboratório Produtivo da Unidade de Ensino de Currais Novos-RN.

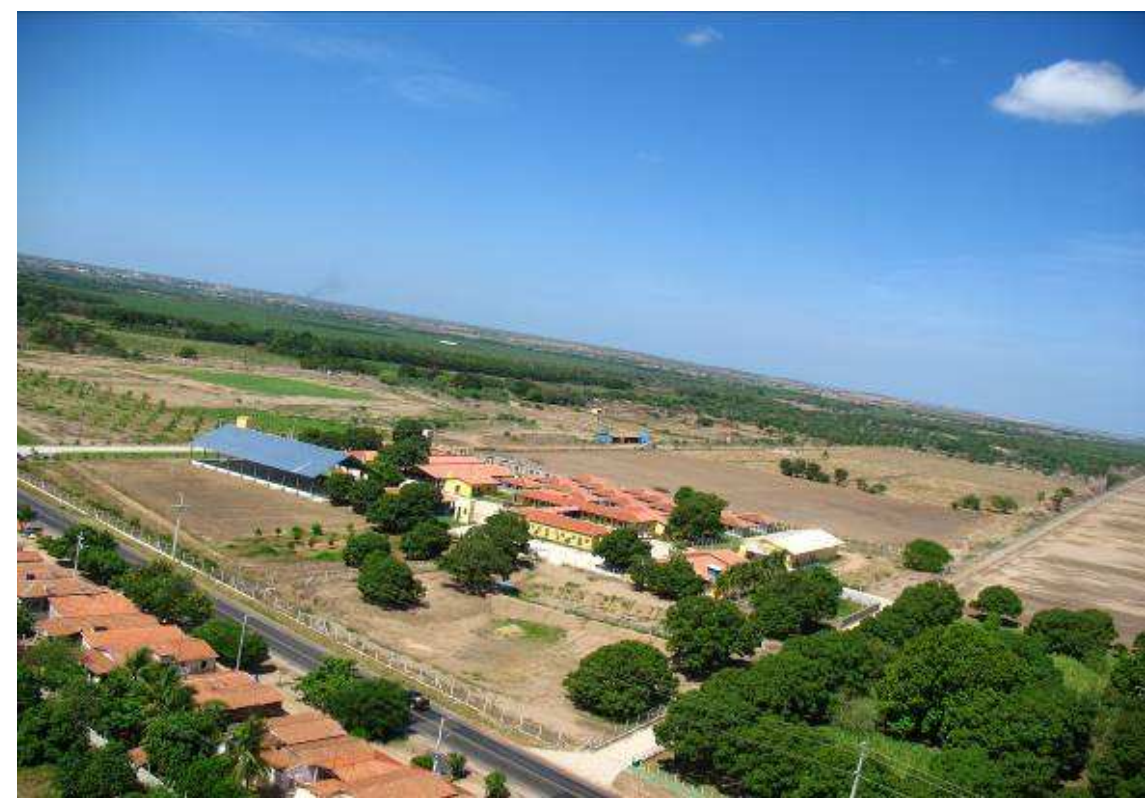

Figura 3 - Vista aérea da Unidade de Ensino de Ipanguaçu-RN. 


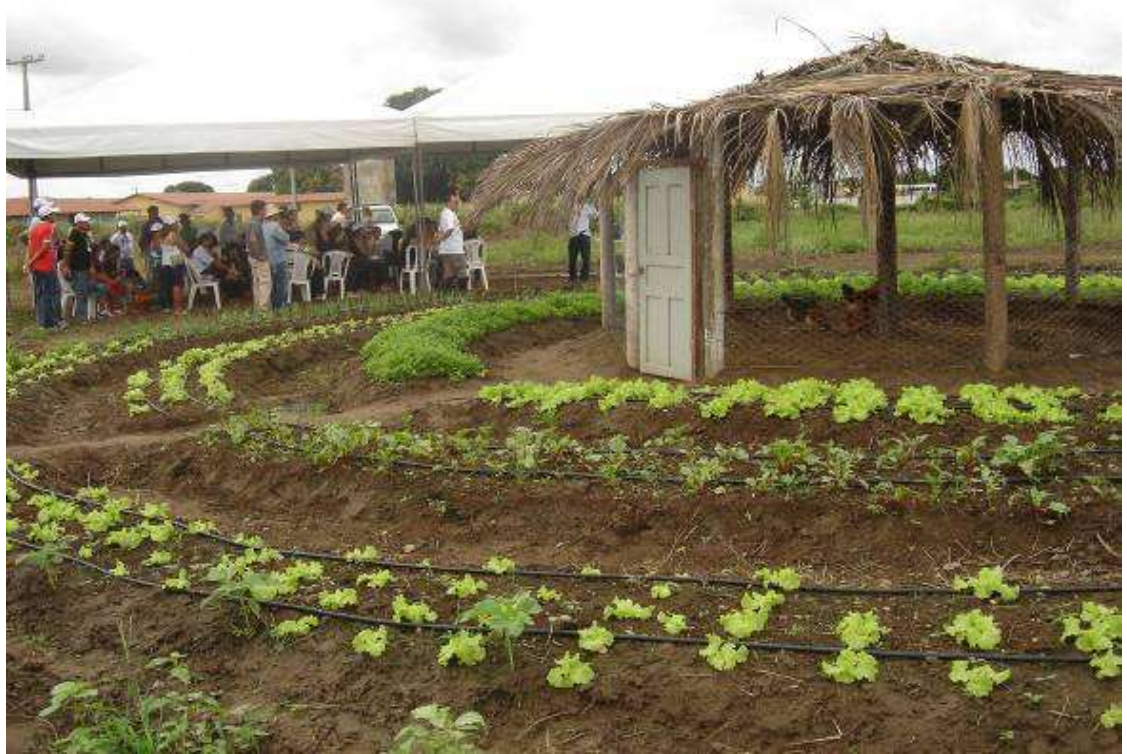

Figura 4 - Atividade agroecológica desenvolvida pelos alunos na Unidade de Ensino de Ipanguaçu-RN.

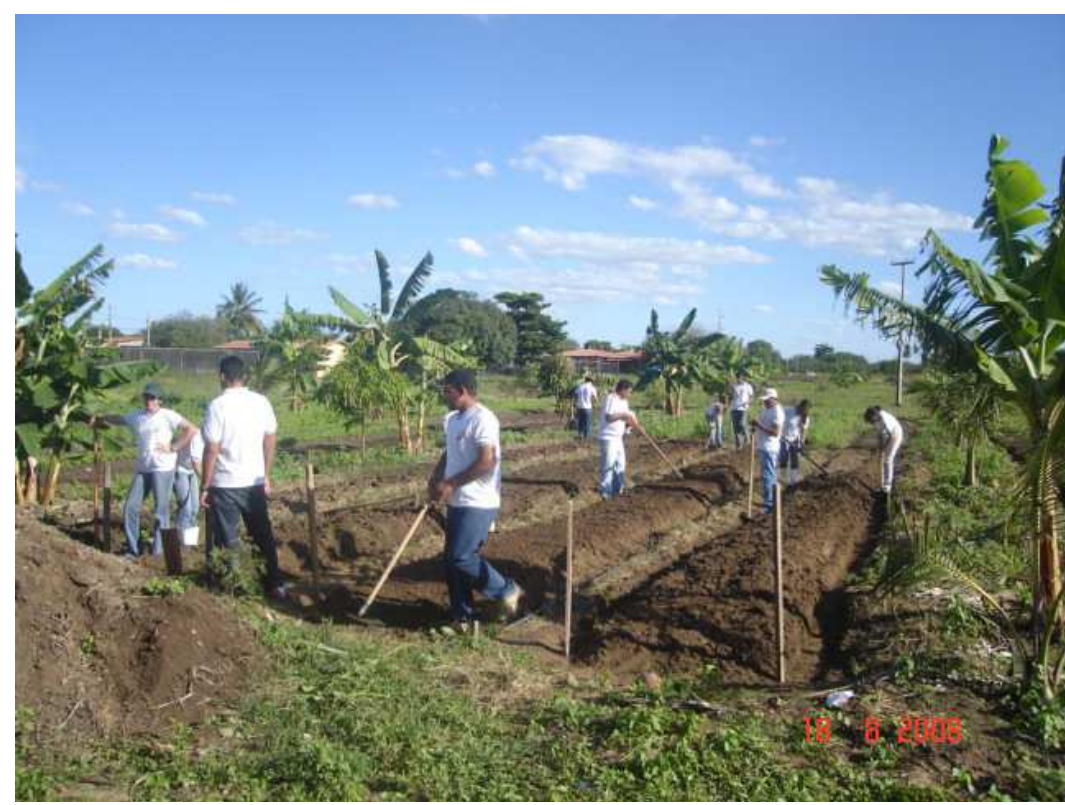

Figura 5 - Desenvolvimento de aula na Unidade de Ensino de Ipanguaçu-RN.

As Unidades de Ensino acima retratadas, vinculadas ao CEFET-RN, demonstram que a consolidação das ações de formação profissional e tecnológica está associada à definição de áreas específicas focadas nas características de desenvolvimento regional. Nesses casos, em estreita articulação com as potencialidades regionais, tem-se o foco nas áreas de laticínios e mineração em Currais Novos e de agroecologia em Ipanguaçu. Tais especificidades aperfeiçoarão as Unidades para liderar o desenvolvimento na área foco e atender as demandas sociais em educação, ciência e tecnologia para a região.

A par disso, a nova realidade, de uma ampla expansão, por si só, já confere à Rede Federal de Educação Profissional e Tecnológica um cenário de experimentos e estudos capaz de 
referendar profundas mudanças na atuação das instituições que a compõem, tendo em vista que estas precisam se estruturar, de forma que, também com a interiorização, venham garantir o cumprimento de sua função social, constituindo-se num ambiente educacional de qualidade adequado à plena formação dos educandos. Nesse contexto, surge a proposta dos Institutos Federais de Educação, Ciência e Tecnologia. A Diretoria de Políticas da Secretaria de Educação Profissional e Tecnológica do Ministério da Educação associa dois sentidos políticos à criação dos Institutos Federais, sendo um relacionado à expansão da rede e o outro à concepção de educação profissional e tecnológica em curso:

"A expansão da Rede Federal de Educação Profissional e Tecnológica, cujo critério na Fase II toma como base a identificação de cidades-pólo, elevará a contribuição da rede federal no desenvolvimento sócio-econômico do país e concorrerá, sobretudo com a interiorização, para uma mais justa ordenação da oferta de EPT, ao incluir locais historicamente postos à margem das políticas públicas voltadas para esta modalidade. Ao estabelecer que todas as unidades vinculadas aos Institutos Federais (inclusive as novas) têm elevado e isonômico grau de autonomia, afirma o território como uma dimensão essencial a sua função e insere na pauta regimental dessas instituições o seu compromisso com um desenvolvimento sócio-econômico que perceba antes o seu 'lócus'. Isto implica uma atuação permanentemente articulada e contextualizada a sua região de abrangência. A autonomia dos campi dos Institutos Federais responde à necessidade de se forjar e fomentar o desenvolvimento de uma Educação Profissional e Tecnológica (Pública) a partir de uma demanda que seja socialmente plena, que considere as diversas representações sociais, desde as oriundas da chamada produção elaborada (grandes firmas), os médios e pequenos empreendimentos e os movimentos sociais. É, pois, função precípua dos Institutos Federais atuar a favor dos arranjos produtivos, sociais e culturais locais. $O$ que está em curso afirma uma concepção de Educação Profissional e Tecnológica como potencializadora do indivíduo no desenvolvimento de sua capacidade de gerar conhecimento a partir de uma prática interativa com a realidade em lugar de uma outra que toma a EPT apenas como modalidade instrumentalizadora do ser humano. Assim, a implantação dos IFET guarda estrito vínculo com o objetivo de desenvolvimento de uma educação profissional cidadã, comprometida com a construção de um país mais digno e ético, uma educação que alcance diferentes grupos e espaços sociais." (PEREIRA, 2008, p. 2-3).

Como resultado do disposto no item 6.2 da Chamada Pública MEC/SETEC nº. 02/2007, de 12 de dezembro de 2007, o Governo Federal divulgou a relação de propostas aprovadas com vistas à constituição dos Institutos Federais de Educação, Ciência e Tecnologia, compreendendo 38 Institutos Federais: Acre, Alagoas, Amapá, Amazonas, Bahia, Baiano, Brasília, Ceará, Espírito Santo, Goiás, Goiano, Maranhão, Minas Gerais, Norte de Minas Gerais, Sudeste de Minas Gerais, Sul de Minas Gerais, Triângulo Mineiro, Mato Grosso, Mato Grosso do Sul, Pará, Paraíba, Pernambuco, Sertão Pernambucano, Piauí, Paraná, 
Fluminense, Rio de Janeiro, Rio Grande do Norte, Rondônia, Roraima, Rio Grande do Sul, Sul Rio-Grandense, Farroupilha, Santa Catarina, Catarinense, Sergipe, São Paulo e Tocantins.

Esses Institutos Federais caracterizam-se por uma estrutura multicampi com proposta de autonomia administrativa e pedagógica, devendo garantir, em sua ação acadêmica, em cada exercício, o mínimo de 50\% das vagas para cursos técnicos de nível médio, bem como o mínimo de $20 \%$ para cursos de licenciaturas e/ou programas especiais de formação pedagógica,visando à formação de professores para a educação básica e para a educação profissional. Os Institutos Federais se constituem a partir da integração de várias unidades educacionais: uns são compostos por uma autarquia com várias unidades subordinadas; outros, por várias autarquias ou por autarquias e unidades subordinadas. Associadas a dificuldades próprias de gestão, algumas das quais decorrentes de distorções históricas, tal caracterização representa um imenso desafio, que, antes de tudo, impõe um repensar quanto à organização estrutural da rede federal. Contudo, ao invés de representar ameaça ou conflitos, essa situação deve ser avaliada e trabalhada como oportunidade para correção e ajustes, de modo a proporcionar a todos os Institutos Federais um ambiente administrativo de equilíbrio, adequado às dimensões assumidas por cada instituição.

Nesse sentido, a elaboração deste texto tem como objetivo suscitar uma reflexão sobre a organicidade administrativa das instituições que compõem a Rede Federal de Educação Profissional e Tecnológica, a partir da realidade atualmente vivenciada, visando contribuir para o debate sobre essa questão.

\section{A REDE FEDERAL E SUA REORGANIZAÇÃO}

No plano nacional, a partir do exercício de 2009, a Rede Federal de Educação Profissional, Científica e Tecnológica fica graficamente representada (Figura 6) pela atuação de referência dos 38 Institutos Federais, supervisionados pelo Ministério da Educação, através da Secretaria de Educação Profissional e Tecnológica. Além dessas instituições, cabe ressalvar, continua também pertencendo à Rede, a Universidade Tecnológica Federal do Paraná, os Centros Federais de Educação Tecnológica Celso Suckow da Fonseca e o de Minas Gerais, além de 24 Escolas Técnicas Vinculadas às Universidades Federais. 


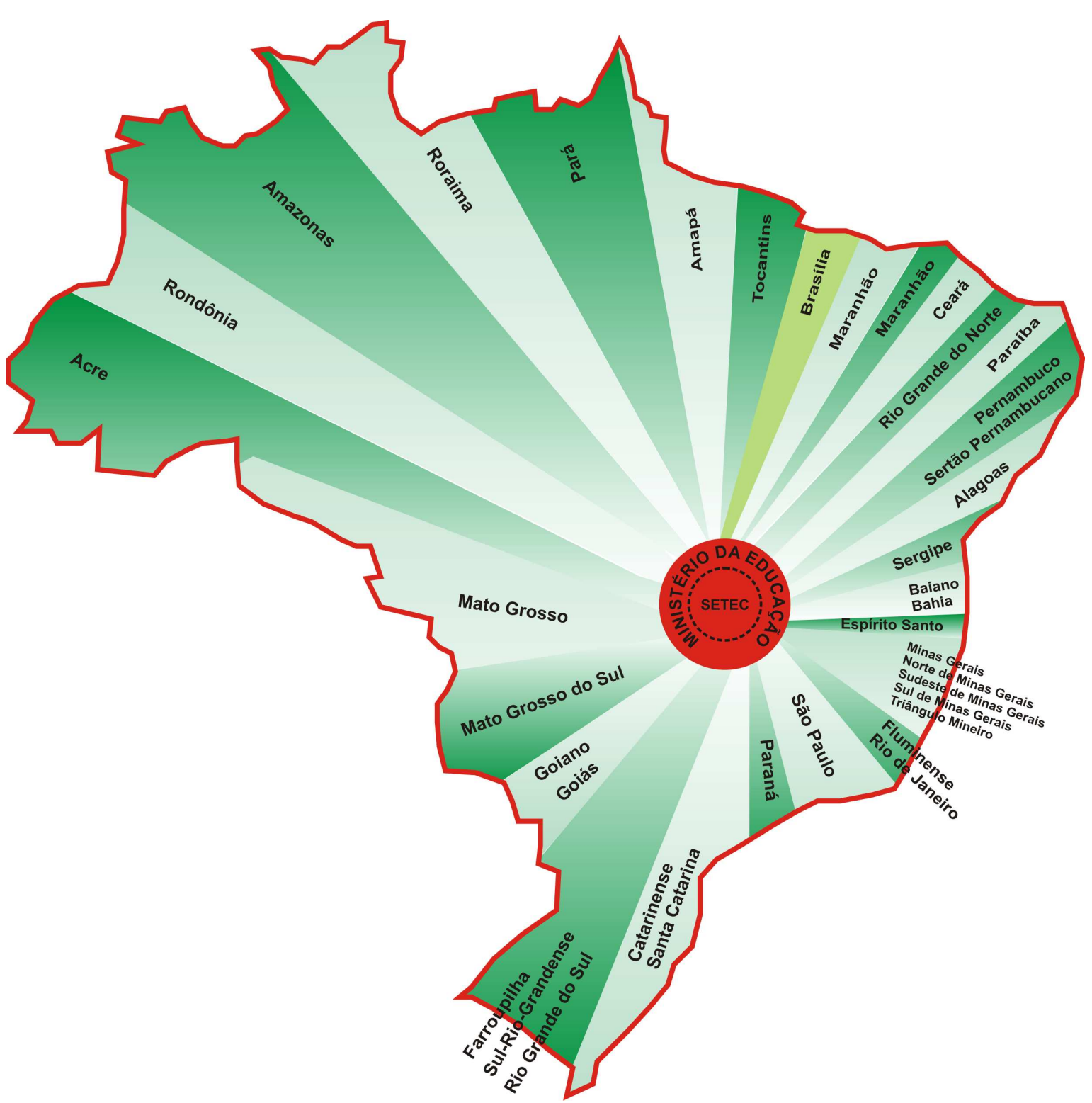

Figura 6 - Representação gráfica da distribuição dos Institutos Federais no país.

No plano interno dos Institutos Federais, um grande desafio é a superação da visão segmentada e/ou setorizada decorrente de modelos de estruturas hierarquizadas em vários níveis. É importante avançar para o que Chiavenato (1999, p. 396) define como "abordagens contemporâneas" para atender às necessidades das organizações: "a utilização de redes e equipes". Concretamente, os Institutos Federais apresentam características de uma organização composta de várias organizações, sendo adequada na gestão dos mesmos, a aplicação de uma estruturação sistêmica. Para Tachizawa (1999, p. 56):

"A adoção do enfoque sistêmico permite que a instituição de ensino analise o meio ambiente e defina o cenário provável, de longo prazo, a partir do qual se delineiam objetivos institucionais e respectivas estratégias para atingi-los. Posteriormente, são identificados processos sistêmicos-chave, indispensáveis para dar suporte a tal delineamento estratégico. Com isso, criam-se condições para estabelecer/revisar a configuração organizacional, os recursos humanos e demais recursos, necessários ao atingimento dos 
objetivos estratégicos. Em seguida, identificam-se as tecnologias da informação e sistemas/software que darão suporte à infra-estrutura organizacional estabelecida."

Nessa condição de enfoque sistêmico, o Instituto Federal passa a ser um conjunto de Unidades de Ensino com gestão delegada e interdependente entre os Campi e a Reitoria. Cada Campus, cumprindo os objetivos finalísticos do Instituto Federal, deve atender princípios de ensino verticalizado, garantido por unidade acadêmica com atuação em todos os níveis da oferta de ensino: educação continuada, cursos técnicos, graduação e pósgraduação; pesquisa, presente em todo o trajeto da formação acadêmica, integrada à verticalização do ensino; relações de extensão e de interação Instituto-Sociedade, também integradas à verticalização do ensino, como um mecanismo de interação do conhecimento científico-tecnológico e de ações sócio-culturais com a sociedade e com o mundo do trabalho; e apoio estudantil, atividade assumida como instrumento de inclusão, acompanhamento e manutenção dos estudantes na escola.

À Reitoria como órgão executivo do Instituto Federal, cabe-lhe a administração, coordenação e supervisão de todas as atividades da Autarquia. Para apoiar, organizar, assistir, coordenar, fomentar e articular a sua ação política e administrativa, deverá contar com um Gabinete, com uma Ouvidoria, órgãos de Auditoria Interna e Consultoria Jurídica e com Assessorias nas áreas de acompanhamento e desenvolvimento de projetos, comunicação social e eventos, informações e dados institucionais.

Compõem ainda a Reitoria, como órgãos executivos, com o objetivo de planejar, superintender, coordenar, fomentar e fiscalizar atividades, cinco Pró-Reitorias, a saber: de Desenvolvimento Institucional - para políticas de desenvolvimento e articulação entre os Campi; de Ensino - para políticas de ensino, articuladas à pesquisa e à extensão; de Extensão e Integração Instituto-Sociedade - para políticas de extensão e relações com a sociedade, articuladas ao ensino e à pesquisa, junto aos diversos segmentos sociais; de Pesquisa e Inovação - para políticas de pesquisa, integrada ao ensino e à extensão, bem como para a promoção de ações de intercâmbio e captação de recursos com instituições e empresas na área de fomento à pesquisa, ciência e tecnologia e inovação tecnológica; e de Planejamento e Administração - para políticas de planejamento, administração, gestão orçamentária, financeira e patrimonial. Além disso, conta com cinco Diretorias Sistêmicas nas seguintes áreas: apoio às atividades estudantis, gestão da tecnologia da informação, gestão de pessoas, engenharia e infraestrutura e educação à distância.

Na realidade, o arranjo situacional dos Institutos Federais é novo e caracteriza-se como próprio de uma estrutura em rede, para integrar sistemicamente diversas organizações de ensino por meio de um núcleo central - no âmbito nacional, através da Secretaria de Educação Profissional e Tecnológica do Ministério da Educação; e no espaço regional, os vários Campi interligados através de uma Reitoria. Com base nesse modelo estratégico, cabe a construção das ações táticas e operacionais para garantir o funcionamento e a função-controle vinculada aos Institutos Federais em todo o território nacional e regionalmente.

Para Morin (2000, p. 90):

"A estratégia deve prevalecer sobre o programa. O programa estabelece uma sequência de ações que devem ser executadas sem 
variação em um ambiente estável, mas, se houver modificação das condições externas, bloqueia-se o programa. A estratégia, ao contrário, elabora um cenário de ação que examina as certezas e as incertezas da situação, as probabilidades, as improbabilidades. $O$ cenário pode e deve ser modificado de acordo com as informações recolhidas, os acasos, contratempos ou boas oportunidades encontradas ao longo do caminho."

Territorialmente, no Instituto Federal, à Reitoria competirá a função estratégica de definição de políticas, supervisão e controle. Para tanto, necessita de uma estrutura administrativa que congregue, além do gabinete, pró-reitorias e diretorias de atuação sistêmica, cabendo a esses órgãos a função de trabalhar matricialmente vinculados às unidades afins dos Campi. Cada Campus, por sua vez, responsável pela execução dos objetivos finalísticos institucionais, necessita de uma estruturação híbrida, através da associação da departamentalização funcional e a matricial - para viabilizar o diálogo e interação dos departamentos da área acadêmica com as unidades operacionais dos demais departamentos das áreas de administração, de apoio ao ensino, de extensão e de pesquisa. O efetivo tamanho das estruturas administrativas dos Campi, dentre os 354 espalhados por todo o país, conforme previsto na meta governamental, terão naturalmente padrões e dimensões diferenciados, em função das especificidades e nível de atuação de cada Campus.

\section{CONSIDERAÇÕES FINAIS}

A implementação da proposta do Governo Lula de expansão e reorganização da Rede Federal de Educação Profissional e Tecnológica, através da criação de 38 Institutos Federais de Educação, Ciência e Tecnologia, exige do Ministério da Educação uma atenção toda especial no tocante ao processo de gestão que será desenvolvido no âmbito interno de cada Instituição e no âmbito nacional, em função da identidade necessária e das diferenças culturais que essas instituições naturalmente incorporarão à nova realidade.

A elaboração deste artigo teve como objetivo provocar uma reflexão sobre a gestão na Rede Federal associada a sua reorganização. Através de análises e debates, talvez as idéias aqui expostas sejam alteradas ou descartadas, o que representa que o texto cumpriu sua função, qual seja a de apresentar uma proposição objetiva para a organicidade dessas instituições, objetivando minimizar as dificuldades ou garantir a eficácia da gestão dos Institutos Federais.

\section{REFERÊNCIAS BIBLIOGRÁFICAS}

BRASIL. Ministério da Educação. Educação Profissional e Tecnológica. Disponível na internet. http://portal.mec.gov.br/setec. Acesso em 02/05/2008.

BRASIL. Ministério da Educação. Instituto Federal - Concepção e Diretrizes. Brasília, 2008.

CHIAVENATO, Idalberto. Administração nos novos tempos. Rio de Janeiro: Campus, 1999. 
FERNANDES, Francisco das Chagas de Mariz. Estrutura e funcionamento dos Centros Federais de Educação Tecnológica. Trabalho apresentado na XXX REDITEC, FortalezaCE, 2006, mimeo.

MORIN, Edgard. Os sete saberes necessários à educação do futuro. São Paulo: Cortez; Brasília, DF: UNESCO, 2000.

PEREIRA, Luiz Augusto Caldas. Institutos Federais de Educação Ciência e Tecnologia.Disponível na internet: http://portal.mec.gov.br/setec/arquivos/pdf3/artigos ifet_jornal.pdf. Acesso em 02/05/2008.

TACHIZAWA, Takeshy e ANDRADE, Rui Otávio Bernardes de. Gestão de instituições de ensino. Rio de Janeiro : Fundação Getúlio Vargas, 1999. 